\title{
Endovascular Treatment for Ruptured Distal Anterior Inferior Cerebellar Artery Aneurysm -Case Report-
}

\author{
Daizo ISHII, Akihiko TAKECHI, \\ Katsuhiro SHINAGAWA, and Takashi SOGABE \\ Department of Neurosurgery, Matsuyama Red Cross Hospital, Matsuyama, Ehime
}

\begin{abstract}
A 73-year-old woman presented with subarachnoid hemorrhage caused by a ruptured left distal anterior inferior cerebellar artery (AICA) aneurysm. Computed tomography showed a thin subarachnoid hemorrhage in the ambient cistern, and digital subtraction angiography revealed an aneurysm arising from the lateral branch of the left AICA, which was separate from the meatal loop. Endovascular treatment was performed to achieve parent artery occlusion using two Guglielmi detachable coils. Postoperatively, the patient had no complications except for left hearing disturbance, and she was independent in daily life. Endovascular parent artery occlusion for distal AICA aneurysm, especially distal from the meatal loop, can avoid sacrificing the internal auditory artery if the lateral branch of the AICA could be occluded more distally from the meatal loop. Sufficient collateral circulation prevents major infarction, and this strategy may be the first-line treatment choice.
\end{abstract}

Key words: distal anterior inferior cerebellar artery, aneurysm, subarachnoid hemorrhage, endovascular treatment, parent artery occlusion

\section{Introduction}

Distal anterior inferior cerebellar artery (AICA) aneurysms are extremely rare, and account for only $0.1 \%$ of all cerebral aneurysms. ${ }^{12)}$ In particular, only 12 cases $^{1-5,7-12)}$ of AICA aneurysm distal to the meatal loop have been reported, and $4^{3,4,9,12)}$ of these were treated by endovascular embolization to occlude the parent artery, with no new complications after the procedure. We describe another case of AICA aneurysm distal to the meatal loop treated by endovascular parent artery occlusion.

\section{Case Report}

A 73-year-old woman, who was being treated for hypertension and diabetes and had a cardiac pacemaker, was referred to our department for severe headache in March 2009. She was drowsy, and computed tomography (CT) showed Fisher group 2 subarachnoid hemorrhage in the ambient cistern (Fig. 1). Her neurological status was Hunt and Hess grade 4, and World Federation of Neurosurgical Societies grade IV. There was no evidence of head injury. Digital subtraction angiography revealed an aneurysm arising from the lateral branch of the left AICA, which was separate from the meatal loop (Fig. 2). The neck of the aneurysm could not be detected. Blood laboratory examinations on admission were unremarkable, and no inflam-

Received June 29, 2009; Accepted October 9, 2009

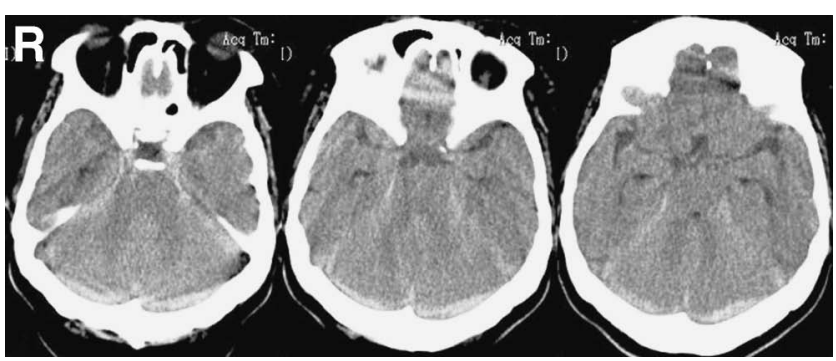

Fig. 1 Computed tomography scans showing a thin subarachnoid hemorrhage in the ambient cistern, but no apparent intraventricular or intracranial hemorrhage.

matory response had developed. Echocardiography detected no evidence of infective endocarditis.

A microcatheter (Excelsior ${ }^{\circledR}$ SL-10 Microcatheter; Boston Scientific, Natick, Mass., U.S.A.) was inserted into the left posterior inferior cerebellar artery (PICA), but the aneurysm was not visualized. Subsequently, the microcatheter was inserted into the left AICA, and the aneurysm was identified (Fig. 3). Despite superselective catheterization, the aneurysm neck was indistinct. Some type of fusiform aneurysm or pseudoaneurysm was suspected, but histological examination was necessary for confirmation. However, given the patient's age, past history (such as the cardiac pacemaker), and her poor neurological status, endovascular treatment to occlude the parent 

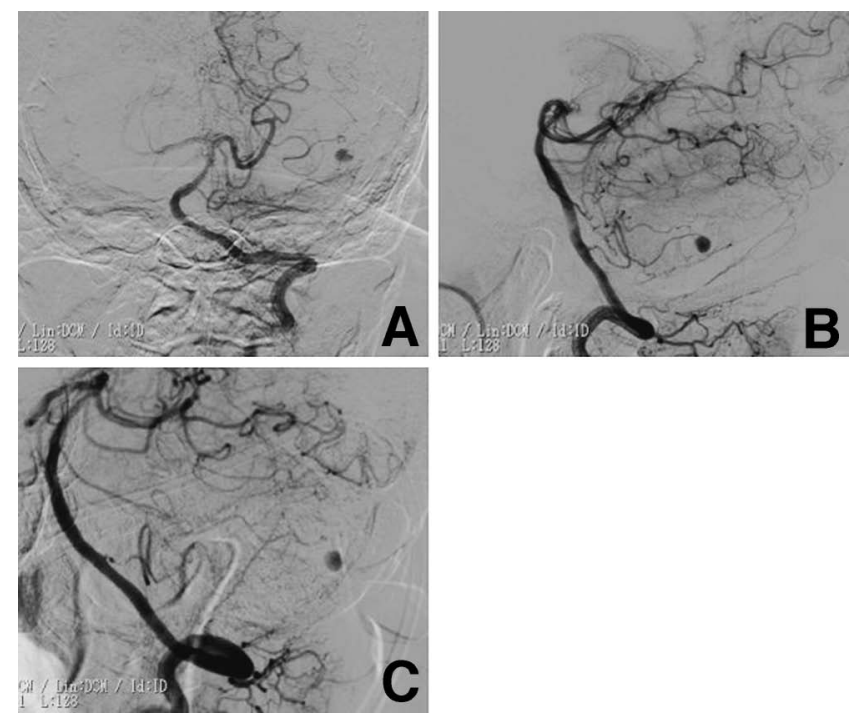

Fig. 2 Digital subtraction angiograms of the left vertebral artery, anteroposterior (A), lateral (B), and left anterior oblique views (C), showing an aneurysm of the left anterior inferior cerebellar artery, separate from the meatal loop.

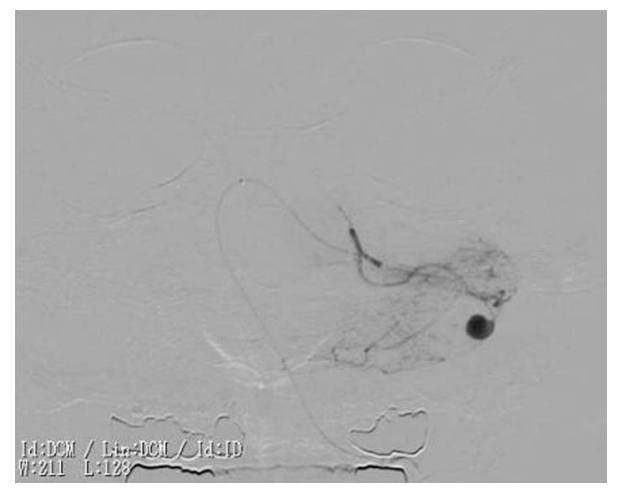

Fig. 3 Superselective angiogram, anteroposterior view, showing the distal anterior inferior cerebellar artery aneurysm, but the neck is not detected.

artery was chosen. The microcatheter was inserted more distally into the lateral branch of the left AICA, passing through the meatal loop, and Guglielmi detachable coils (GDC $\otimes$; Boston Scientific) were deployed. The first coil (GDC UltraSoft Coil: $2 \mathrm{~mm} \times 2 \mathrm{~cm}$; Boston Scientific) was detached, but the coil was not entwined. The next coil was entangled with the first coil, the microcatheter was pulled back to near the meatal loop, and the second coil (GDC UltraSoft Coil: $2 \mathrm{~mm} \times 3 \mathrm{~cm}$; Boston Scientific) was deployed at that position. Complete embolization of the lateral branch of the left AICA was successfully achieved. Final left vertebral angiography demonstrated invisibility of the aneurysm (Fig. 4).

Postoperatively, the patient was alert, with no complications except for left hearing disturbance, which was highgrade, sensorineural hearing loss. No otolaryngologic
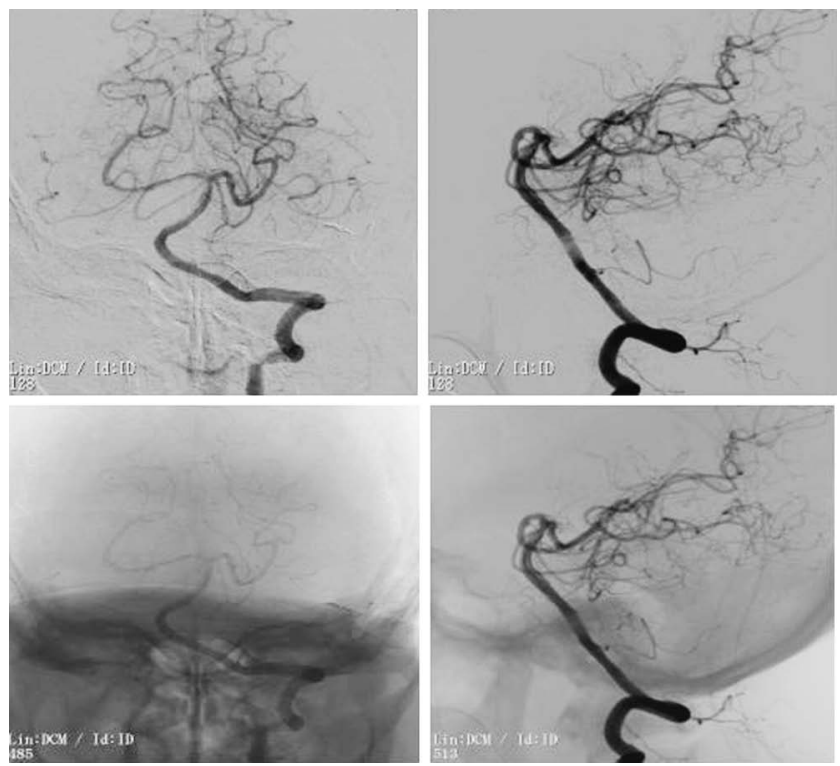

Fig. 4 Upper row: Post-procedure left vertebral subtraction angiograms, anteroposterior (left) and lateral views (right), showing the invisibility of the aneurysm. Lower row: Left vertebral non-subtraction angiograms, anteroposterior (left) and lateral views (right).

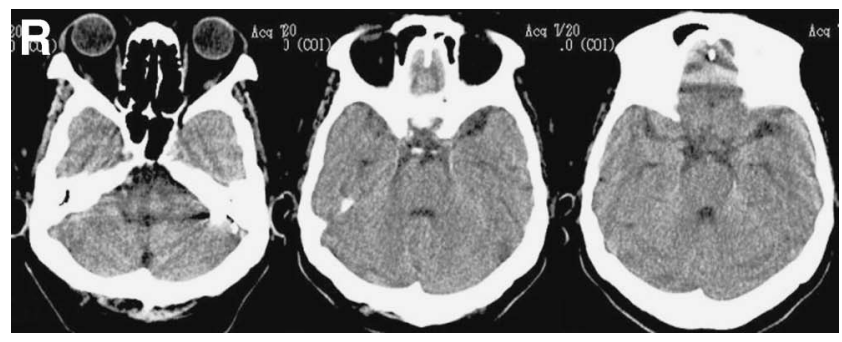

Fig. 5 Computed tomography scans showing no apparent infarction after the operation.

problems were identified in her left tympanum or inner ear. Therefore, decreased flow of the left internal auditory artery after the AICA embolization was suspected. Magnetic resonance (MR) imaging could not be performed because of her cardiac pacemaker. CT showed no apparent infarction (Fig. 5). Furthermore, CT did not clarify whether cerebellar or brain stem infarction had occurred because of the coil artifact. However, the patient was independent in her postoperative activities of daily living.

\section{Discussion}

Table 1 summarizes the 12 reported cases ${ }^{1-5,7-12)}$ of distal AICA aneurysms that were separate from the meatal loop. All patients presented with subarachnoid hemorrhage, intraventricular hemorrhage, and/or intracranial hemorrhage. Three patients ${ }^{7,9,11)}$ had complications, such as cranial nerve palsy, from the onset, and their symptoms did not recover after the procedure. Only five of the de- 
Table 1 Summary of anterior inferior cerebellar artery (AICA) aneurysms distal to the meatal loop

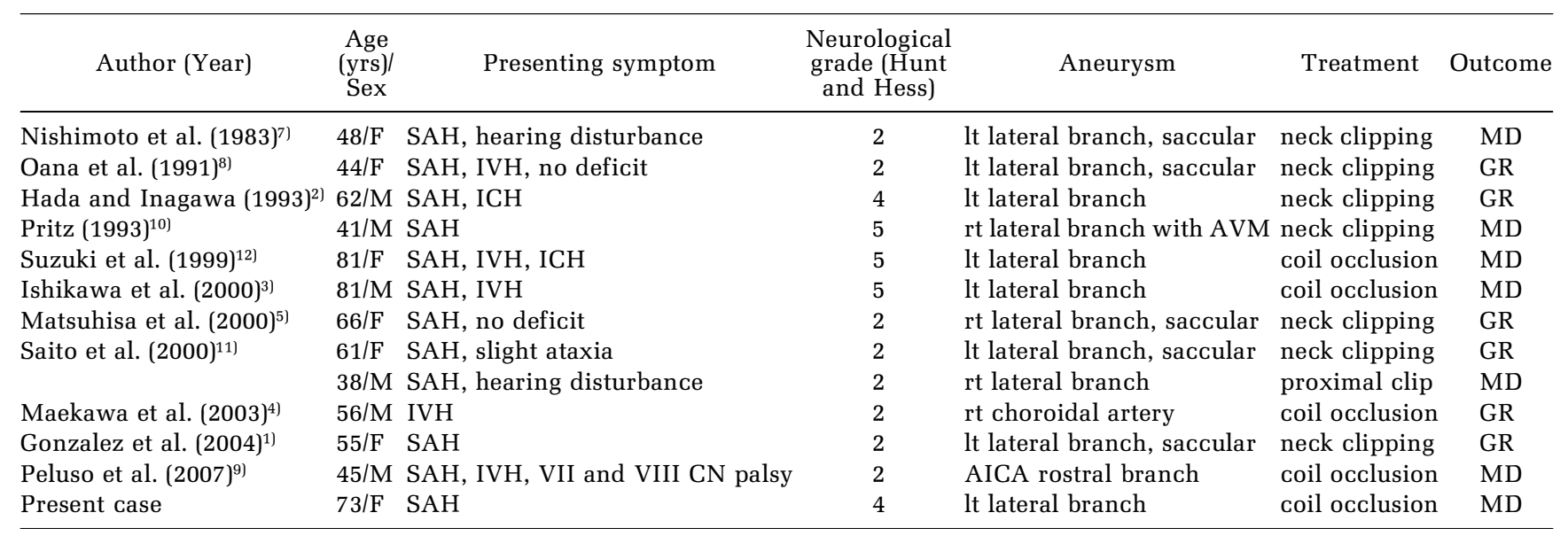

AVM: arteriovenous malformation, CN: cranial nerve, GR: good recovery, ICH: intracranial hemorrhage, IVH: intraventricular hemorrhage, MD: moderately disabled, SAH: subarachnoid hemorrhage.

scribed aneurysms $s^{1,5,7,8,11)}$ were saccular. The AICA has congenital vulnerability, so distal AICA aneurysms appear to occur at both divergences but also at elbows. ${ }^{11)}$ However, this series contained few cases. Given that only 5 of the 13 cases were saccular aneurysms, distal AICA aneurysms may tend to be wide necked or fusiform.

Seven cases $^{1,2,5,7-10)}$ were treated by neck clipping, five ${ }^{3,4,9,12)}$ by endovascular treatment, and one ${ }^{11)}$ by proximal clipping. All endovascular treatments were performed to occlude the parent artery. The aneurysms were located in the distal AICA, so a microcatheter was difficult to lead into the aneurysm. No deterioration occurred after the coil occlusion in four cases. Only in the present case did hearing disturbance become evident after the procedure. Left vertebral angiography 10 days after the operation revealed that the flow of the left AICA was preserved from the vertebral artery to near the meatal loop, as found by the final left vertebral angiography at the operation. Cilostazol was prescribed ( $200 \mathrm{mg} /$ day) immediately after the operation as antithrombotic therapy. Therefore, retrograde thrombosis to the stem of the left AICA after the embolization was not suspected. Of course, the acoustic nerve palsy may have occurred at the onset, but the internal auditory artery was probably sacrificed after the embolization. The first coil stretched when it was detached. Therefore, the microcatheter had to be pulled back to near the meatal loop to entangle the second coil with the first coil. Consequently, we had no choice but to deploy the second coil near the meatal loop. If a microcatheter could be inserted more distally in the AICA, the flow of the internal auditory artery could possibly be preserved. However, the present patient suffered no other complications after the procedure. Hearing disturbance occurred or deteriorated postoperatively after clipping treatment in only one case, and occurred with embolization treatment in our case. However, there is probably no significant difference in the incidence of hearing disturbance between clipping and embolization treatments.

MR imaging revealed cerebellar infarction post-occlu- sion in only one case, ${ }^{4)}$ but the clinical consequences were limited, and the clinical outcome was good recovery. The AICA has many anastomoses with the superior cerebellar artery and PICA. ${ }^{12)}$ Occlusion of the lateral branch of the AICA, especially distal to the meatal loop, is unlikely to cause a major infarction in the brain stem or cerebellum. In fact, complications of such an infarction would be limited because of the many anastomoses of the AICA.

To investigate the cause of a peripheral aneurysm, histological examination of the aneurysm is necessary to determine any evidence of head injury or infection, such as infective endocarditis. The most common mechanism for the formation of distal cerebellar artery aneurysms is arterial dissection caused either by local trauma or nonspecific inflammation.6) In the distal cerebellar arteries, the morphology and possible higher hemodynamic stresses could contribute to dissection and subsequent aneurysm formation. ${ }^{6)}$ In the present case, no histological examination was performed, although the aneurysm may have been a dissecting aneurysm. Distal AICA aneurysms are often wide necked or fusiform, and the neck of the aneurysm may be difficult to clip. Endovascular treatment to achieve parent artery occlusion is the first-line treatment choice for an AICA aneurysm distal to the meatal loop, considering the patient's age and neurological condition.

\section{References}

1) Gonzalez LF, Alexander MJ, McDougall CG, Spetzler RF: Anteroinferior cerebellar artery aneurysms: surgical approaches and outcomes-a review of 34 cases. Neurosurgery 55: 1025-1035, 2004

2) Hada $H$, Inagawa $T$ : [A case of ruptured distal anterior inferior cerebellar artery aneurysm]. Jpn J Stroke 15: 323-327, 1993 (Jpn, with Eng abstract)

3) Ishikawa E, Yanaka K, Meguro K, Narushima K, Suzuki K, Wada M, Nose T: [Treatment of peripheral aneurysms of the posterior circulation]. No Shinkei Geka 28: 337-343, 2000 
(Jpn, with Eng abstract)

4) Maekawa M, Awaya S, Fukuda S, Teramoto A: [A ruptured choroidal artery aneurysm of the anterior inferior cerebellar artery obliterated via the endovascular approach: case report]. No Shinkei Geka 31: 523-527, 2003 (Jpn, with Eng abstract)

5) Matsuhisa T, Teramachi H, Hirata T: [A case of ruptured aneurysm of the distal anterior inferior cerebellar artery]. Surgery for Cerebral Stroke 28: 56-59, 2000 (Jpn, with Eng abstract)

6) Mitsos AP, Corkill RA, Lalloo S, Kuker W, Byrne JV: Idiopathic aneurysms of distal cerebellar arteries: endovascular treatment after rupture. Neuroradiology 50: 161-170, 2008

7) Nishimoto A, Fujimoto S, Tsuchimoto S, Matsumoto $Y$, Tabuchi K, Higashi T: Anterior inferior cerebellar artery aneurysm. Report of three cases. J Neurosurg 59: 697-702, 1983

8) Oana K, Murakami T, Beppu T, Yamaura A, Kanaya H: Aneurysm of the distal anterior inferior cerebellar artery unrelated to the cerebellopontine angle: case report. Neurosur- gery 28: 899-903, 1991

9) Peluso JP, van Rooij WJ, Sluzewski M, Beute GN: Distal aneurysms of cerebellar arteries: incidence, clinical presentation, and outcome of endovascular parent vessel occlusion. AJNR Am J Neuroradiol 28: 1573-1578, 2007

10) Pritz MB: Aneurysms of the anterior inferior cerebellar artery. Acta Neurochir (Wien) 120: 12-19, 1993

11) Saito A, Ezura M, Takahashi A, Yoshimoto T: [An arterial dissection of the distal anterior inferior cerebellar artery treated by endovascular therapy]. No Shinkei Geka 28: 269-274, 2000 (Jpn, with Eng abstract)

12) Suzuki K, Meguro K, Wada M, Fujita K, Nose T: Embolization of a ruptured aneurysm of the distal anterior inferior cerebellar artery: case report and review of the literature. Surg Neurol 51: 509-512, 1999

Address reprint requests to: Daizo Ishii, M.D., Department of Neurosurgery, Matsuyama Red Cross Hospital, 1 Bunkyo-cho, Matsuyama, Ehime 790-8524, Japan.

e-mail: zokun@xd6.so-net.ne.jp 